\title{
Influencia de las cuatro fases lunares en la fenología y producción en las tres variedades de alfalfa (medicago sativa) en el distrito de Vilcabamba- Grau Apurímac 2014-2015
}

He influences of the four lunar phases in her fenología and production in the three
alfalfa varieties (medicago sativa) in Vilcabamba's district - Grau Apurímac 2014-2015

\author{
Cirilo Mario Ccaira Mamani \\ mariocaira1966@gmail.com - Gerente "Panificadora caira" \\ https://orcid.org/0000-0003-1027-0218 \\ Alex Ernesto Muñoz Cáceres \\ alexmuca@hotmail.com - Universidad Nacional Micaela Bastidas \\ https://orcid.org/0000-0002-0238-3362 \\ Alcides Torres Cutire \\ torrescuterialcides@gmail.com - Universidad Nacional Micaela Bastidas \\ Julio Rolando Blas Quispe \\ blasjulior@gmail.com - Universidad Nacional Micaela Bastidas \\ https://orcid.org/0000-0002-3796-4730
}

Recibido el 19/12/20 | Aceptado el 31/12/20

DOI: https://doi.org/10.47190/nric.v3i1.134

\section{Resumen}

El objetivo: La Influencia de las fases lunares en la fenología y producción en tres variedades de alfalfa (Medicago sativa). Método: Se consideró el experimental y con el diseño factorial, factores de estudios "A" las fases lunares y "B" variedades de alfalfa. Resultados: Se obtuvieron la cosecha después de establecimiento del promedio de 70 días, Tabla 1. La producción materia verde, por cortes y días, el siguiente: 35 y 44 días dependiendo de la estación del año. Tabla 2. Análisis de variancia de la materia verde en 190 días, existen el nivel de significancia entre las fases lunares y las variedades de alfalfa $F_{c}=14.824, F_{c}=76.7698 ; F_{\infty}=3.05, F_{\infty}=3.44$, y el coeficiente de la variabilidad de $11.33 \%$. En la tabla 3 . Se evalúa la media de rango múltiple de significancia de Tukey (0.01) en las fases lunares de la producción de materia verde del cultivo de alfalfa; se llegó a las siguientes conclusiones: primer lugar la Luna Cuarto Creciente con de $5,732.6401 \mathrm{Kg} / \mathrm{há}$; segundo lugar la Luna Cuarto Menguante con 4,857.5098 kg/há; tercer lugar la Luna Nueva con 4,639.6499kg/há; y por último la Luna Llena con una producción de 4,042.73 kg/há. Tabla 4 Efecto de las variedades (Factor de estudio "B"). En la prueba de significancia de Tukey en la variedad Cuf 101, fue superior de las demás con una producción de 6, 277.14 kg/há, segundo lugar está la variedad Moapa con 4,643.55 kg/há, y con menor producción de materia verde es la variedad W 350 con $3,533.68$ kg/há.

Palabras claves: Fases lunares-Variedades del cultivo de alfalfa.

Como citar: Ccaira-Mamani, C.M., Muñoz-Caceres, A.E., Torres Cutire, A. \& Blas Quispe, J.R. (2021). Influencia de las cuatro fases lunares en la fenología y producción en las tres variedades de alfalfa (medicago sativa) en el distrito de Vilcabamba- Grau Apurímac 2014-2015. ÑAWPARISUN - Revista de Investigación Científica, 3(2), 19-25. 


\begin{abstract}
The objective: The influence of the lunar phases in her fenología and production in three varieties of alfalfa (Medicago sativa). Method: He considered himself the experimental and with the design factorial, studies factors To the lunar phases and B alfalfa varieties. Aftermaths: They obtained the harvest of the after establishment of the average of 70 days, Tabla 1. The production green matter, for cuts and days, the following: 35 and 44 days depending on the year's season. Board 2. variancia's Analysis of the green matter in 190 days, exist significancia's level among the lunar phases and the alfalfa varieties Fc; F8 $=3.05, F 8=3.44$, and the coefficient of 11.33 variability. In the board 3 . He evaluates the of rank his stocking multiple of Tukey's significancia $(0.01)$ in the lunar phases of the green- matter production of the alfalfa cultivation; following conclusions were reached: First place the Luna First quarter with of $5,732.6401 \mathrm{Kg} / \mathrm{há}$; Second place the Luna Last quarter with 4,857.5098 kg/há; Third place the New moon with 4,639.6499 kg/há; And finally the Full moon with 4,042.73 kg/há's production. Board the varieties's 4 Efecto ( study Factor B ). In significancia's test of Tukey in the variety Cuf 101, he was superior of them besides with a production of $6,277.14 \mathrm{~kg} / \mathrm{há}$, second place is the variety Moapa with $4,643.55 \mathrm{~kg} / \mathrm{há}$, and green- matter production is with minor the variety W 350 with $3,533.68 \mathrm{~kg} / \mathrm{há}$.
\end{abstract}

Keywords: Lunar Phases - Varieties of the alfalfa cultivation.

\section{Introducción}

La civilización comenzó cuando en lugar de cazar, el hombre creó los animales de los que se alimentaba y cultivó las plantas que necesitaba. Así nacieron la agricultura y la ganadería. De este modo, el hombre tenía más garantizada su alimentación, pero eso también permitió que aumentara su población (Parramón, 2005, p.82).

Tomas Robert Malthus. Sostiene que la población aumenta en progresión geométrica mientras que los medios de sustancia lo hacen aritmética, planteando así la necesidad de controlar por cualquier medio el crecimiento poblacional, principalmente de los trabajadores (Siccha,2001, p. 41). En Europa estaba teniendo lugar una amplia revolución industrial que transformaba el continente: de ser una sociedad agraria pasaría a ser una sociedad industrial. Esto provocó un fuerte movimiento de crecimiento demográfico y económico de las ciudades. El crecimiento de la población llegó a una dimensión no conocida hasta entonces, a pesar de que millones habían abandonado el continente en dirección a América y por la gran hambruna de la papa y la miseria social (De Soto,2007, p.68).

El incremento en las eficiencias en la productividad agrícola, en el mejoramiento de plantas y animales, en las labores de mecanización y otras técnicas de manejo, han sido factores importantes en mantener el balance de equilibrio entre la oferta de alimentos y la demanda mundial de los mismos. Sin embargo, el adecuado abastecimiento de nutrientes esenciales para los cultivos, y seguirá siendo el enlace crítico entre la producción de alimentos para satisfacer las necesidades de actuales y el sostenimiento agrícola a largo plazo (Mamani, 2011, pp.154-155)
La alfalfa está hoy prácticamente extendida por todo el mundo. Sin embargo, dada la gran variedad de ecotipos existentes en el estado espontáneo en la región, se fija su área de origen en Asia Menor y sur del Cáucaso (De Candolle, 1919), abarcando esta zona geográfica Turquía, Siria, Irán, Afganistán, parte occidental de Pakistán y Cachemira. De aquí es probable que se extiende su cultivo a Grecia, como consecuencia de las guerras médicas (aproximadamente 470 años a. de C.). Serian, pues, los griegos quienes le dieron el nombre de médica, que recogido por los romanos se ha conservado hasta nuestros días como denominación de su género botánico (Del Pozo, 1983, pp. 23-24)

La alfalfa tiene su área de origen en Asia Menor y sur del Cáucaso, abarcando países como Turquía, Irán, Siria, Afganistán y Pakistán. La alfalfa, por su calidad como forrajera, su alta productividad y los aportes a la conservación del suelo, es unas especies que el productor puede considerar en su planteo productivo. (Sánchez, 2005, p.96)

Utilización de pasturas de alfalfa en la Sierra. Una vez establecido el cultivo de alfalfa sola o asociada con Dactylis glomerata que se logra a los seis meses después de siembra, el primer corte o pastoreo ligero (la mediados de mayo) se realiza cuando el cultivo ha alcanzado un buen tamaño de crecimiento de 25 a $30 \mathrm{~cm}$ de altura. Después del año de establecimiento, los pastoreos o cortes subsiguientes tendrán que hacerse cada 36 a 54 días dependiendo de la variedad y estación del año. (Choque, 2005, pp. 120-121).

Variedades de alfalfa: La variedad Moapa, se desarrolla en las zonas ecológicas Valles interandinos 2400 a 2800, a una dominancia Intermedia, densidad de siembra 30 
$\mathrm{Kg} / \mathrm{Ha}$, rendimiento de materia verde 132.2 $\mathrm{TM} / \mathrm{Ha} / \mathrm{Año} \mathrm{(Choque,2005);} \mathrm{la} \mathrm{alfalfa} \mathrm{de} \mathrm{la}$ variedad Cuf 101 fue desarrollada por la Universidad de California, USA, y en nuestro país es una variedad pública que se ha difundido por todo nuestro territorio. Fue uno de los primeros cultivares en ser introducidos y en la actualidad sigue siendo una de las variedades con el área sembrada más extensa, a pesar de haber sido superada técnicamente por otras variedades. La cuf 101 es de grupo 9, esto significa que el periodo que deja de crecer durante el invierno es muy corto. Es tolerante al pulgón verde y azul, de latencia invernal corta, de corona pequeña. Apta para henificar y de buena producción de forraje. Es susceptible a enfermedades de hoja (Hortus,2006, p. 12); variedad W350 tiene la dormancia de 3.8, la producción de 15 a 20 años, rendimiento 100 Tm/há al año, es un forraje que aporta $24 \%$ de proteínas, vitamina, fósforo, potasio, cobre, hierro y nitrógeno (Choque, 2005, p. 208).

\section{Claudio Ptolomeo (87-150 d. C.)}

Este filósofo, matemático y astrónomo griego nació y vivió en Alejandría durante el siglo II a. C. Escribió una monumental obra en trece tomos, Sintaxis matemática, donde recogió todos los conocimientos de astronomía que tienen hasta esa época. Además, realizó tablas astronómicas y una importante obra de cartografía que sirvió para elaborar los mapas más exactos de su época. También realizó un catálogo de estrellas que recoge un total de 1,200 . Su principal aportación a esta ciencia es el modelo planetario que creó y que describió en cinco libros. Su concepto del universo se impuso en la astronomía y perduró durante más de trece siglos (Parramón, 2006.p 71)

La cristiandad tuvo pocos problemas modelo geocéntrico ptolemaico, que dejaba espacio en el universo, más allá de las estrellas fijas, para acomodar un cielo y un infierno de manera que la Iglesia adoptó este modelo del universo como una verdad establecida (Hawking,2004, p.16).

\section{Nicolás Copérnico (1473-1543)}

Astrónomo polaco que nació en Torun, a orillas de Vistula, el 19 de febrero de 1473, murió en Frombrock el 24 de mayo de 1543. Estudió leyes, astronomía y lenguas en las universidades de Cracovia Bolonia y Padua. Desde 1512 fue canónigo en Frombrock, dedicándose entonces a las labores de su cargo y a las observaciones astronómicos. También realizó algunos inventos muy útiles para la ciudad, como un sistema hidráulico que permitía el suministro de agua. Fue un cuidadoso analista de todas las teorías conocidas hasta entonces y las comparó con los datos más recientes disponibles y con sus propias observaciones. Todo eso le hizo llegar a la conclusión de que la tierra no era el centro del universo (Parramòn, 2006, p. 72).

\section{Sir Isaac Newton (1642-1727)}

Nació el día de Navidad en Woolsthorpe, Inglaterra, realizó el más grande de los aportes, para explicar por qué giran los planetas en torno al Sol.

La ley de gravitación universal, considera que todo cuerpo del Universo atrae a otro cuerpo con una fuerza que es directamente proporcional al producto de las masas de ambas partículas e inversamente proporcional al cuadrado de la distancia que los separa. (Hawking, 2004, pp.127-159).

El más notable físico y matemático de los siglos XVII y XVIII fue Isaac Newton, que vio la primera luz en Inglaterra en 1643. Su visión del Universo estremeció todo el edificio de la ciencia y la filosofía de su tiempo, formulando las leyes fundamentales de la mecánica y de la gravedad universal; interpretó las leyes de Juan Kepler en términos de aceleración centrípeta, por lo que dedujo que la fuerza entre la Tierra y su satélite la Luna es inversamente proporcional al cuadrado de la distancia. (Núñez, 1998, pp. 166-168).

\section{La Luna.}

La Luna, el astro más próximo a nosotros, ha fascinado siempre a los hombres, ya sean poetas o científicos. $Y$ ha desempeñado un importante papel en la historia de la humanidad. En la antigüedad proporcionó al hombre un calendario natural: la sucesión regular de sus fases permitió definir una cronología de los acontecimientos y medir unas duraciones. Newton descubrió la Ley de la gravitación mediante la observación del movimiento de revolución de la Luna alrededor de la Tierra. Al igual que la Tierra, la Luna gira sobre si misma; esta rotación dura 28 días, y es igual a la duración del movimiento de traslación de la Luna alrededor de la Tierra. Este sincronismo entre los movimientos de rotación y de traslación es consecuencia de la atracción gravitacional de la Tierra sobre la Luna. Pero la Luna ejerce también una atracción gravitacional sobre la Tierra (Schoeller, 1985, p.46).

La Tierra tiene un satélite, la Luna, que se encuentra a unos 350,00-400,000 km de distancia. La Luna describe alrededor de la Tierra una órbita elíptica en un tiempo de 28 días y medio. Este tiempo se denomina periodo de lunación o mes lunar. Simultáneamente, la Luna gira sobre sí misma, tardando también 28 días y medio en completar su giro. A esta coincidencia temporal entre los dos movimientos lunares se debe que desde la Tierra veamos siempre la misma cara de nuestro satélite. La cara oculta de la Luna no fue 
conocida hasta que las naves espaciales consiguieron fotografiarla. Las fases de la Luna son: Luna nueva, cuarto creciente, cuarto menguante, Luna llena (La Republica, 2003, pp. 16-17).

Las fases de la Luna. La Luna se mueve alrededor de la tierra en el mismo sentido de giro que nuestro planeta. Tarda en completar la rotación unos 29 días, quedando o no iluminada por el Sol en cada una de las posiciones de su órbita. Cuando la cara visible está iluminada por el Sol decimos que es la fase de luna llena, mientras que cuando el Sol ilumina la cara no visible, decimos que está en fase de luna nueva. Las fases de cuarto menguante y cuarto creciente son cunadas sólo se ve la mitad de la superficie (Parramón, 2006, p.55).

\section{MATERIAL Y MÉTODOS}

El presente trabajo de investigación se realizó el 28 diciembre de 2014 a julio 2015, en la Universidad Nacional Micaela Bastidas- Sede Vilcabamba de la Facultad de Ingeniería Agroecológica y Desarrollo Rural, de la Escuela Académico Profesional Ingeniería Agroecología y Desarrollo Rural, en el Distrito Vilcabamba.

La parcela experimental fue constituida por $3.24 \mathrm{~m}^{2}$, en un área neta total de $116,64 \mathrm{~m}^{2}$. La variedad de alfalfa que se aplicó son los siguientes: Meticago sativa Moapa, Medicago sativa W350 y Medicago sativa Cup 101 (Mostacero, 2002). La instalación de la primera siembre se realizó el 28 de diciembre 2014 en la fase lunar cuarto creciente, segunda siembra 04 de enero 2015 en la fase luna llena; tercera siembra 13 de enero, cuarta siembra 20 de enero (Brisol,2015, pp. 4-24).

\section{MÉTODO:}

Se empleó el diseño experimental de Bloque Completo al Azar con arreglo factorial de $4 \times 3 x$ 3 , el factor " $A$ " constituido por las fases lunares y el factor "B" por tres variedades del cultivo de alfalfa: Moapa, W 350 y Cuf 101, en tres repeticiones.

Se uso para los cálculos, un nivel de significancia del 0.5. El análisis estadístico usando el ANOVA (Vásquez,1990, p.129), utilizando el software SPSS (Statistical Package for Social Sciences), con lo cual obtuvieron coeficiente de variabilidad. Prueba de rangos múltiples de Ducan (Rodríguez, 2000. P.86). Estas pruebas estadísticas sirvieron para diferenciar la producción en la influencia de las fases lunares.

\section{RESULTADOS}

La tabla 1. Comparamos el promedio días de corte y producción de materia verde del cultivo de alfalfa; en el primer corte de materia verde el promedio de 69 días; primer lugar la variedad CUP101 de 7,150.00 kg/ha; en el segundo la variedad Moapa de $6,240 \mathrm{~kg} / \mathrm{ha}$, en el tercer con $3,810.00 \mathrm{~kg} / \mathrm{ha}$. Segundo corte de materia verde a los 38 días: primer lugar la variedad Moapa con 4,930.00 kg/ha; en segundo la variedad W350 con 4,100.00 kg/ha; en el tercer la variedad CUP101 con 3,190.00 kg/ha. Tercer corte de materia verde el promedia a 35 días, la variedad CUP101 primer lugar con 8,220.00 $\mathrm{kg} / \mathrm{ha}$; en el segundo ocupa la variedad Moapa con 3,660.00 kg7ha; en tercer, la variedad W350 con 2,690.00 kg/ha. En el cuarto corte de materia verde con promedio de 44 días, la CUP101 con 6,640.00, Moapa con 3740.00 y W350 con $3530 \mathrm{~kg} / \mathrm{ha}$, respectivamente.

La tabla 2. Análisis de ANOVA; obtuvimos el coeficiente de variabilidad de 11.33, el valor está dentro del parámetro permisible. El factor de estudio " $A$ " las fases lunares existe significancia $\mathrm{Fc}=14.824 \mathrm{Ft}=3.05$; el factor de estudio "B" las variedades de alfalfa, existen significancia de $\mathrm{FC}=76.7698$ y $\mathrm{Ft}=3.44$. Efectos de bloque no existe diferencia estadística entre los bloques, nos indica que se comportó en forma homogénea.

Tabla 3. Efecto de las fases lunares (Factor de estudio "A"). Se evalúa la media de rango múltiple de significancia de Tukey (0.01) en las fases lunares de la producción de materia verde del cultivo de alfalfa; se llegó a las siguientes conclusiones: primer lugar la Luna Cuarto Creciente con la producción de materia verde de 5,732.6401 Kg/há; segundo lugar la Luna Cuarto Menguante con la producción de materia verde de 4,857.5098 kg/há; tercer lugar se ubica la Luna Nueva con una producción de materia verde 4,639.6499 kg/há; y por último la Luna Llena con una producción de materia verde 4,042.73 kg/há

Tabla 4 Efecto de las variedades (Factor de estudio "B"). En la prueba de significancia de Tukey en la variedad Cuf 101, fue superior de las demás con una producción de 6, 277.14 $\mathrm{kg} /$ há, segundo lugar está la variedad Moapa con 4,643.55 kg/há, y con menor producción de materia verde es la variedad W 350 con $3,533.68$ kg/há

Tabla 5 observamos los siguientes resultados de la interacción entre las fases lunares y la variedad de alfalfa, de los tratamientos siguientes : T11 Luna Cuarto Menguante con variedad Cuf101, con la producción de 8224.2197 kg/há; T09 Luna Cuarto Creciente con variedad Cuf101, con producción de 7146.8701 kg/há; T12 Luna Nueva con variedad 
Cuf101 con producción de 6641.5698 kg/há; T01 Luna Cuarto Creciente con variedad Moapa con producción de 6242.73 kg/há; T02 Luna Llena con variedad Moapa con producción de 4927.46 kg/há; T06 Luna Llena con variedad de W350 con producción de 4104.8301 kg/há; T05 Luna Cuarto Creciente con W350 con producción de $3808.3201 \mathrm{Kg} / \mathrm{há}$; T04 Luna Nueva con variedad de W350 con producción de $3743.95 \mathrm{Kg} / \mathrm{h}\{\mathrm{a} ;$ T03 Luna Cuarto Menguante con variedad Moapa con producción de 3660.8 kg/há; T08 Luna Nueva con variedad W350 con producción de 3533.3301 kg/há; T10 Luna Llena con variedad Cuf101 con producción de 3095.8899 kg/há; T07 Luna Cuarto Menguante con variedad W350 2688.23 kg/há.

Tabla 1.

Producción materia verde de alfalfa, por cortes y días.

\begin{tabular}{|c|c|c|c|c|}
\hline VARIEDADES & COSECHAS & $\mathrm{Kg} / \mathrm{m} 2$ & Kg/Há & $\bar{X}$ DÍAS \\
\hline \multirow{4}{*}{ MOAPA } & Corte $^{10}$ & 0,624 & 6240.00 & 40 \\
\hline & Corte $2^{\circ}$ & 0,493 & 4930.00 & 38 \\
\hline & Corte $3^{\circ}$ & 0,366 & 3660.00 & 35 \\
\hline & Corte $4^{\circ}$ & 0,374 & 3740.00 & 44 \\
\hline \multirow{4}{*}{ W350 } & Corte $1^{\circ}$ & 0,381 & 3810.00 & 40 \\
\hline & Corte $2^{\circ}$ & 0,410 & 4100.00 & 38 \\
\hline & Corte $3^{\circ}$ & 0,269 & 2690.00 & 35 \\
\hline & Corte $4^{\circ}$ & 0,353 & 3530.00 & 44 \\
\hline \multirow{4}{*}{ CUP101 } & Corte $1^{\circ}$ & 0,715 & 7150.00 & 40 \\
\hline & Corte $2^{\circ}$ & 0,319 & 3190.00 & 38 \\
\hline & Corte $3^{\circ}$ & 0,822 & 8220.00 & 35 \\
\hline & Corte $4^{\circ}$ & 0,664 & 6640.00 & 44 \\
\hline
\end{tabular}

Fuente: Escuela Profesional Ingeniería Agroecología y desarrollo Rural (UNAMBA)-Apurímac.

Tabla 2.

Análisis de variancia de la materia verde de alfalfa y las fases lunares en 190 días.

\begin{tabular}{|c|c|c|c|c|c|c|}
\hline FV & $\mathrm{GL}$ & SC & $\mathrm{CM}$ & $F$ & $\mathrm{~F} \infty=0.05$ & SIG \\
\hline REPETICIONES & 2 & 2189504 & 1094752 & 3.6774 & 3.44 & * \\
\hline FACTOR “A” & 3 & 13239104 & 4413034.5 & 14.824 & 3.05 & ** \\
\hline FACTOR "B" & 2 & 45708096 & 22854048 & 76.7698 & 3.44 & ** \\
\hline INTERACCION & 6 & 47755904 & 7959317.5 & 26.7364 & 2.55 & ** \\
\hline ERROR & 22 & 6549312 & 297696 & & & \\
\hline TOTAL & 35 & 115441920 & & & & \\
\hline
\end{tabular}

Fuente: Escuela Profesional Ingeniería Agroecología y desarrollo Rural (UNAMBA)-Apurímac.

Coeficiente de variabilidad, es de $11.33 \%$, buena

Tabla 3.

Evaluación media de rango múltiple de significancia de Tukey (0.01) en las fases lunares en la producción materia verde en alfalfa.

\begin{tabular}{llcc}
\hline & FACTOR "A" & MEDIA & SIG \\
\hline LCC & LUNA CUARTO CRECIENTE & 5732.6401 & $\mathrm{a}$ \\
LM & LUNA CUARTO MENGUANTE & 4857.5098 & $\mathrm{~b}$ \\
LN & LUNA NUEVA & 4639.6499 & $\mathrm{bc}$ \\
LLL & LUNA LLENA & 4042.73 & $\mathrm{c}$ \\
\hline FU
\end{tabular}

Fuente: Escuela Profesional Ingeniería Agroecología y desarrollo Rural (UNAMBA)-Apurímac.

Tabla 4.

Evaluación de media de la prueba de rango múltiple de Tukey $(p=0,01)$ para variedades de alfalfa en la producción de materia verde.

\begin{tabular}{clcc}
\hline FACTOR "B" Variedades de alfalfa & MEDIA & \\
\hline V3 & Variedad Cuf 101 & 6277.14 & a \\
V1 & Variedad Moapa & 4643.55 & b \\
V2 & Variedad W 350 & 3533.68 & $\mathrm{c}$ \\
\hline
\end{tabular}

Fuente: Escuela Profesional Ingeniería Agroecología y desarrollo Rural (UNAMBA)-Apurímac. 
Prueba de rango múltiple de Tukey $(\mathrm{p}=0.01)$, la interacción entre las fases lunares y las variedades de alfalfa.

\begin{tabular}{clrc}
\hline TRATA. & & MEDIA & $\mathrm{Kg} / \mathrm{há}$ \\
\hline T11 & LUNA CUARTO MENG X CUF101 & 8224.2197 & $\mathrm{a}$ \\
T09 & (LUNA CCUARTO CRE X CUF101 & 7146.8701 & $\mathrm{ab}$ \\
T12 & (LUNA NUEVA X CUF101 & 6641.5698 & $\mathrm{ab}$ \\
T01 & (LUNA CUARTO CRE X MOAPA & 6242.73 & $\mathrm{~b}$ \\
T02 & LUNA LLENA X MOAPA & 4927.46 & $\mathrm{c}$ \\
T06 & (LUNA LLENA X W350 & 4104.8301 & $\mathrm{c}$ \\
T05 & (LUNA CUART CRECIEN X W350 & 3808.3201 & $\mathrm{c}$ \\
T04 & (LUNA NUEVA XW350 & 3743.95 & $\mathrm{c}$ \\
T03 & LUNA CUARTO MENG X MOAPA & 3660.8 & $\mathrm{c}$ \\
T08 & LUNA NUEVA X W350 & 3533.3301 & $\mathrm{c}$ \\
T10 & LUNA LLENA X CUF101 & 3095.8899 & $\mathrm{c}$ \\
T07 & LUNA CUARTO MENGU X W350 & 2688.23 & $\mathrm{c}$ \\
\hline
\end{tabular}

Fuente: Escuela Profesional Ingeniería Agroecología y desarrollo Rural (UNAMBA)-Apurímac.

\section{DISCUSIÓN}

El análisis de resultado se fundamenta: el ritmo anual del Sol ascendente y descendente. A lo largo del año, el Sol se desplaza por la noria cósmica inclinada. Se observa que cuando sube, la primavera y el verano se manifiestan sobre la Tierra en su atmósfera vivificada, mientras que su descenso provoca como respuesta una vitalización de los suelos, subsuelos y raíces. Las respiraciones mensuales de la luna son vividas del mismo modo por la Tierra. Por lo que respecta al Sol, manifiesta una magistral respiración anual que es el origen de las estaciones de la Tierra.

Por otro lado, el Sol en su periplo pasa por delante de cada una de las 12 constelaciones zodiacales, resultado de lo cual son los 12 meses del calendario. (Florin, 2016, p.29).

Recolección de forrajes. Respecto a la modalidad de cosechar en verde para alimento inmediato del ganado, se recomiendan los cortes en Luna Llena, dentro de los límites que podrá permitirse la explotación agropecuaria. En esta fase las plantas poseen un máximo de poder nutritivo, además de conseguir eliminar en buena parte el peligro de recalentamiento, tan perjudicial para el ganado. (Farrerons, 2010, p. 47).

Efectos de las Luna en la agricultura. Luna nueva: Los días de luna nueva se originan los cambios de las fuerzas lunares de abajo hacia arriba. Sin embargo, la savia sigue activa en la parte inferior de las raíces, por lo cual el poder germinativo de las semillas es mínimo. Este aspecto es importante sobre todo para los últimos tres días antes de la luna nueva y el día de luna.

Cuarto creciente. En esta fase lunar la savia sube y despliega sus fuerzas en troncos, tallos, hojas y flores, fomentando el crecimiento, la floración y la maduración. Las mareas son bajas o muertas, el movimiento de los líquidos en los animales comienza a activarse.
Luna llena. En Luna Llena, se despliegan al máximo las fuerzas lunares fomentando el poder germinativo y el crecimiento; sin embargo, también pueden exigir demasiado de las plantas, por lo tanto, no es recomendable realizar labores que afecten directamente las plantas. La savia bruta de las plantas sube dinámicamente hasta las hojas, las mareas son altas y vivas, el movimiento del líquido en los animales es más intenso.

Luna Menguante. La tierra ahora inhala, por lo cual, la savia elaborada va a las raíces de las plantas para desde ahí desplegar su fuerza. El suelo y las plantas absorben más agua y nutrientes. El crecimiento se desacelera y las plantas se fortifican. Ahora es la mejor luna para la mayoría de las labores. Las fuerzas de la luna menguante se potencian al realizar las labores por la tarde (Rosas, 2006, pp. 102-105).

Frijol: la altura no presentó diferencias estadísticas significativas positivas $(P \geq 0.05)$, en relación a la fase lunar; sin embargo es importante resaltar que entre tratamientos, se presentó una diferencia de altura de $1 \mathrm{~m}$ en las plantas sembradas en $\mathrm{CM}$, esto indica que las diferencias estadísticas no fueron significativas, probablemente por una falta de repeticiones. Barreiro (2003) y Restrepo (2005), reportan que las hortalizas que producen frutos aéreos y flores para el consumo, crecen y se desarrollan mejor cuando son sembradas en la fase de CC, sin embargo, los resultados obtenidos no permiten tal aseveración, en el caso particular del frijol. (Fontana, 2014). 


\section{CONCLUSIONES}

En total cortes, de materia verde del cultivo de alfalfa, con lo cual logramos el objetivo específico de la determinación de la mayor influencia de las fases lunares en las fases fenológicas de las variedades de alfalfa. Se muestra en la tabla 1 el mínimo y máximo 35 y 44 días de corte, dependiendo de las estaciones del año.

Tabla 3. Efecto de las fases lunares (Factor de estudio "A"). Se evalúa la media de rango múltiple de significancia de Tukey (0.01) en las fases lunares de la producción de materia verde del cultivo de alfalfa; se llegó a las siguientes conclusiones: primer lugar la Luna Cuarto Creciente con la producción de materia verde de $5,732.6401 \mathrm{Kg} / \mathrm{há}$; segundo lugar la Luna Cuarto Menguante con la producción de materia verde de 4,857.5098 kg/há; tercer lugar se ubica la Luna Nueva con una producción de materia verde 4,639.6499 kg/há; y por último la Luna Llena con una producción de materia verde $4,042.73 \mathrm{~kg} / \mathrm{há}$

Tabla 4 Efecto de las variedades (Factor de estudio "B"). En la prueba de significancia de Tukey en la variedad Cuf 101, fue superior de las demás con una producción de 6, 277.14 kg/há, segundo lugar está la variedad Moapa con 4,643.55 kg/há, y con menor producción de materia verde es la variedad W 350 con 3,533.68 kg/há.

\section{REFERENCIAS BILIOGRÁFICAS}

Biblioteca Hernando de Soto (2015). Los 12 economistas más importantes de la historia. Ediciones segunda Grupo Editorial Norma. Lima Perú. (p. 181)

Bristol. (2014). Almanaque calculado expresamente para la República del Perú. (p.32)

Bristol. (2015). Almanaque calculado expresamente para la República del Perú. (pp. 4-21)

Choque Lázaro Julio (2005). Producción y manejo de especies forrajeras. Ediciones primeras Editorial Universidad Nacional del altiplano. Puno-Perú. (pp. 56-224)

Fontana Fontana L.M.C., M.A. Ruiz, G. Blain, F.J. Babinec \& N.A. R. (2014). Producción y calidad de forraje de alfalfa con gramíneas mega térmica en el año de implementación. Argentina.

Ferrerons. A (2010). Influencia de la Luna en la Agricultura Edición sexta Editorial Grupo Mundi Prensa- Madrid España. (pp. 10-47)

Florin Xavier (2016). Jardinería práctica cultivar en armonía con la Luna y el Cielo. Editorial Susaeta Madrid-España. (pp. 17-31)

Hortus S.A. (2006). Semilla forrajera. La alianza esperada trabajando para la ganadería peruana. Barranca- Perú. (pp. 12-14)

Mamani Mamani Evaristo (2011). Materia orgánica y producción para la agricultura ecológica. Ediciones primera Editorial UNA- (pp.154-187). Puno Perú.

Parramón Ediciones (2006). Atlas visual de astronomía. Ediciones Impreso QUEBECOR WORLD PERÜ S.A. (pp.5571)

Parramón Ediciones S.A. (2006). Atlas visual de ecología. Edición primera Editorial Q.W. S.A.C. (p.16)

Rosas Roa, Antonio (2006). Agricultura orgánica práctica. Edición quinta Editorial Medios Impresos Colombia.

Sánchez Reyes Cristian (2005) Cultivo y producción pastos y forrajes. Ediciones primeras Editorial RiPALMA Lima Perú (pp.96-124) 